\title{
Numerical scalings of the decay lengths in the scrape-off layer
}

\author{
Militello, F.; Naulin, V; Nielsen, Anders Henry
}

Published in:

Plasma Physics and Controlled Fusion

Link to article, DOI:

$10.1088 / 0741-3335 / 55 / 7 / 074010$

Publication date:

2013

Link back to DTU Orbit

Citation (APA):

Militello, F., Naulin, V., \& Nielsen, A. H. (2013). Numerical scalings of the decay lengths in the scrape-off layer. Plasma Physics and Controlled Fusion, 55(7), 074010. https://doi.org/10.1088/0741-3335/55/7/074010

\section{General rights}

Copyright and moral rights for the publications made accessible in the public portal are retained by the authors and/or other copyright owners and it is a condition of accessing publications that users recognise and abide by the legal requirements associated with these rights.

- Users may download and print one copy of any publication from the public portal for the purpose of private study or research.

- You may not further distribute the material or use it for any profit-making activity or commercial gain

- You may freely distribute the URL identifying the publication in the public portal

If you believe that this document breaches copyright please contact us providing details, and we will remove access to the work immediately and investigate your claim. 


\title{
Numerical scalings of the decay lengths in the Scrape-Off Layer
}

\author{
F. Militello, ${ }^{1}$ V. Naulin, ${ }^{2}$ and A.H. Nielsen ${ }^{2}$ \\ ${ }^{1}$ EURATOM/CCFE Fusion Association Culham Science Centre, Abingdon, Oxon, OX14 3DB, UK \\ ${ }^{2}$ Association Euratom DTU, Technical University of Denmark, \\ P.O Box 49, DK 4000 Roskilde, Denmark
}

\begin{abstract}
Numerical simulations of L-mode turbulence in the Scrape-Off Layer (SOL) are used to construct power scaling laws for the characteristic decay lengths of the temperature, density and heat flux at the outer midplane. Most of the results obtained are in qualitative agreement with the experimental observations despite the known limitation of the model. Quantitative agreement is also obtained for some exponents. In particular, an almost linear inverse dependence of the heat flux decay length with the plasma current is recovered. The relative simplicity of the theoretical model used allows to gain insight into the mechanisms determining the width of the Scrape-Off Layer.
\end{abstract}




\section{INTRODUCTION}

One of the major concerns for the next generation tokamaks and for the future fusion reactors is the exhaust of heat and particles [1]. This process controls the plasma flow from the core towards the first walls and the divertor, and determines not only the longevity of the plasma facing components, but also the overall performance of the machine. The physics of the exhaust is regulated by the narrow region of plasma surrounding the well confined core known as the Scrape-Off Layer (SOL). The defining feature of this part of the machine is the fact that here the field lines do not close on themselves, but impinge on solid surfaces.

In this context, it is particularly important to be able to predict how the heat and the particles are deposited on the plasma facing components. Often, these processes are quantitatively described by so called decay lengths, which represent how the relevant profiles fall off beyond the last closed flux surface in cross-field direction. A formal definition of the decay length for the generic quantity $f(x)$, function of the radial coordinate $x$, is $\lambda_{f} \equiv$ $f(x) /(d f(x) / d x)$. Obviously, $\lambda_{f}$ is a scalar number only if the profile of $f$ is exponential while, more generally, $\lambda_{f}$ is a function of $x$.

In addition, the characteristic width of the SOL (which is related to the decay lengths), changes as one moves along the field lines, so that it is narrower at the outer midplane (where the turbulent structures erupt from the core) than in the proximity of the divertor. The former width is relevant, for example, to determine whether the gap between the last closed flux surface and the first wall is sufficient to avoid a strong interaction between the plasma and the solid materials. The latter can be used to calculate the deposition pattern of the heat at the target. Clearly, these widths are related to each other and often the midplane width is chosen as a reference. This is also our choice, since in the remaining of the paper the decay length will always refer to the midplane region.

From an experimental point of view, the upstream width can be measured with reciprocating Langmuir probes while, at the divertor, infra-red thermography can be used to evaluate the deposition of the heat fluxes. Under the assumption that the SOL can be identified with a flux tube, the mid-plane and target decay lengths can be mapped to each other by the flux expansion, which takes into account the magnetic geometry of the machine. Therefore, the mid-plane decay length provides a more amenable quantity for extrapolations as its projection at the target can be generalized to different divertor configurations. 
Due to the presence of open field lines, the width of the SOL is determined by the balance between the parallel and the perpendicular transport. In L-mode, the latter is mainly caused by turbulence, and in particular by coherent structures (called filaments or blobs) generated inside the last closed flux surface and expelled into the SOL. Several experimental observations describe the SOL turbulence as strongly intermittent, non Gaussian and with large fluctuations with respect to the background [2-4]. All these features can be captured by a theoretical framework based on the dynamics of the filaments [5-7]. Numerical simulations based on this approach proved successful in reproducing experimental data in both conventional $[8,9]$ and spherical [10] tokamaks.

In this work, we apply regression analysis in order to extract scaling laws for the decay lengths from a large database of SOL L-mode turbulence simulations. In other words, we apply an experimental method to extract relevant information from the results obtained with our theoretical model. This approach has the benefit of identifying trends with the dimensionless parameters that govern the system. This allows to associate the behaviour of the decay lengths to specific physical mechanisms described in our equations.

In the next Section, we discuss the theoretical model that we used and how the simulations were set up to solve it. In Section III we introduce the results of the regression analysis and we construct the scaling laws. Finally, in Section IV we summarize the work and draw our conclusions.

\section{PHYSICAL MODEL AND ITS NUMERICAL SOLUTION}

\section{A. Equations and definitions}

The model that we use is designed to capture the dynamics of the plasma filaments in the SOL $[7,9,11]$. It consists of three drift-fluid equations which describe the evolution of the normalized density, $n$, electron temperature, $T$, and plasma vorticity, $\Omega$. The equations 
are:

$$
\begin{aligned}
\frac{\partial n}{\partial t}+\frac{1}{B}[\phi, n] & =n C(\phi)-C\left(n T_{e}\right)+D \nabla_{\perp}^{2} n-\Sigma_{n} n \\
\frac{\partial T}{\partial t}+\frac{1}{B}[\phi, T] & =\frac{2}{3} T C(\phi)-\frac{7}{3} T C(T)-\frac{2}{3} \frac{T^{2}}{n} C(n)+\chi \nabla_{\perp}^{2} T-\Sigma_{T} T \\
\frac{\partial \Omega}{\partial t}+\frac{1}{B}[\phi, \Omega] & =-C\left(n T_{e}\right)+\mu \nabla_{\perp}^{2} \Omega-\Sigma_{\Omega} \Omega, \\
\Omega & =\nabla_{\perp}^{2} \phi .
\end{aligned}
$$

Note that Eq.4 relates the vorticity to the electrostatic potential, $\phi$, as the former is calculated using only the $\mathbf{E} \times \mathbf{B}$ velocity. All the equations are dimensionless since use was made of the Bohm normalization, which scales all the lengths with respect to the ion Larmor radius calculated with the electron temperature, $\rho_{s}$, and the time with respect to the inverse of the ion gyro-frequency, $\Omega_{i}$. We remind that $\rho_{s} \equiv c_{s} / \Omega_{i}$ where $c_{s} \equiv \sqrt{T_{0} / m_{i}}$ and $\Omega_{i} \equiv Z e B_{0} / m_{i}\left(m_{i}\right.$ and $Z$ are the ion mass and charge state, $e$ the electron charge, $T_{0}$ a characteristic electron temperature and $B_{0}$ the modulus of the confining magnetic field). Note also that the $\mathbf{E} \times \mathbf{B}$ advection in all the equations is represented with a Poisson bracket notation: $B^{-1}[\phi, f] \equiv B^{-1} \mathbf{b} \times \nabla \phi \cdot \nabla f(f$ here is a generic scalar field). In addition, the magnetic field $B^{-1} \approx 1+\epsilon+\left(\rho_{s} / R\right) x$, where $\epsilon$ is the inverse aspect ratio, $R$ is the major radius and $x$ is the "radial" coordinate

The collisional dissipation is modelled with terms that represent the normalized particle diffusivity, $D$, thermal diffusivity, $\chi$, and viscosity, $\mu$. Their definition, which relies on neoclassical transport, is:

$$
\begin{aligned}
& D=\left(1+1.3 q^{2}\right)(1+\theta) \frac{\rho_{e}^{2} \nu_{e i}}{\rho_{s}^{2} \Omega_{i}} \\
& \chi=\left(1+1.6 q^{2}\right)\left[4.66 \frac{\rho_{e}^{2} \nu_{e e}}{\rho_{s}^{2} \Omega_{i}}+\Theta_{i e} 2 \frac{\rho_{i}^{2} \nu_{i i}}{\rho_{s}^{2} \Omega_{i}}\right] \\
& \mu=\left(1+1.6 q^{2}\right) \frac{3}{4} \frac{\rho_{i}^{2} \nu_{i i}}{\rho_{s}^{2} \Omega_{i}}
\end{aligned}
$$

where $q$ is the safety factor, $\rho_{e}$ and $\rho_{i}$ are the electron and ion Larmor radius, $\nu_{s s^{\prime}}$ is the collision frequency between the species $s$ and the species $s^{\prime}$ (with "i" for the ions and "e" for the electrons), $\theta=T_{0, i} / T_{0, e}, \Theta_{i e} \equiv\left[1+\left(\nu_{e, \epsilon}^{*} / \nu_{e}^{*}\right)^{2}\right]^{-1}$ with the equipartition collisionality $\nu_{e, \epsilon}^{*} \approx 63$ for a deuterium plasma and the collisionality $\nu_{e}^{*} \equiv L / \lambda_{e}$ ( $L$ is the characteristic parallel length scale of the filament, which is related to the mid-plane to target connection length, and $\lambda_{e}$ the electron collisional mean free path). 
The turbulence dynamics is investigated in a drift plane (i.e. perpendicular to the magnetic field lines) located at the outer mid-plane of the machine. The parallel evolution is not described self-consistently and it is partially recovered by introducing ad hoc terms which represent the losses towards the divertor. In the case of the density and of the vorticity, such losses are assumed to have an advective nature, as the filament expands at a fraction of the sound speed in the parallel direction, while the temperature losses are modelled with a conductive approximation. The terms associated with these effects are multiplied by the following factors:

$$
\begin{aligned}
\Sigma_{n} & =\Sigma_{\Omega}=\frac{M_{\|} \xi c_{s}}{L \Omega_{i}} \\
\Sigma_{T} & =\frac{2}{3} \frac{\chi_{\|, e}}{L^{2} \Omega_{i}},
\end{aligned}
$$

where $M_{\|}$is the Mach number of the parallel flows, $\xi \equiv \sqrt{Z+\theta}$ and $\chi_{\|, e}=3.2\left(v_{t e}^{2} / \nu_{e e}\right)(1+$ $\left.4 / \nu_{e}^{*}\right)^{-1}$ is the parallel electron heat conduction, which includes a flux limiter correction for low collisionalities ( $v_{t e}$ is the electron thermal velocity). Note also that the lack of parallel dynamics prevents a proper treatment of the drift-wave, which might play a role in the filament evolution [12].

On the other hand, the model properly captures the interchange mechanism that is thought to be at the base of the perpendicular motion of the filaments in the SOL [6]. In particular, a local slab approximation is used to simplify the curvature terms, which appear in the equations through the operator $C(f) \equiv\left(\rho_{s} / R\right) \partial f / \partial y$, where $f$ is a generic scalar field, $\rho_{s}$ is the ion Larmor radius calculated with the electron temperature, $y$ is the "poloidal" coordinate in the drift plane.

The problem is closed by the definition of the boundary conditions. In the "poloidal" direction, it is natural to assume periodicity. In the "radial" direction, we impose an inner boundary which connects the edge region with the core plasma. Here we fix the temperature and the density so that, in their normalized version, they are $T=1$ and $n=1$ (i.e. $T_{\text {dimensional }}=T_{0}$ ). Similarly, we fix $\Omega=\phi=0$. The outer boundary condition is at the far end of the SOL, where we assume $\Omega=v_{y}=0$ and no heat and particle fluxes, $\partial T / \partial x=$ $\partial n / \partial x=0$ (here $v_{y}=\partial \phi / \partial x$ is the "poloidal" plasma velocity). Both the dissipative and parallel loss terms are kept constant in space and time throughout each simulations and their values are calculated using experimental quantities (i.e. edge temperature, density,...) measured at a position corresponding to the inner boundary of the numerical domain. 
Note that once the boundary conditions are fixed, the behaviour of the system 1-4 depends only on the seven dimensionless parameters that appear in the equations, which are: $\rho_{s} / R$, $\epsilon, D, \chi, \mu, \Sigma_{n}$ and $\Sigma_{T}$. It is therefore clear that also derived quantities, such as the normalized decay lengths, are uniquely determined by these parameters. This suggests that it is possible to identify power law scalings relating the SOL width to the dimensionless parameters $[13-16]$.

\section{B. Numerical set up}

Equations 1-4 are numerically solved with the ESEL code [7], which has a history of successful applications to filament dynamics studies $[7,17]$ as well as to experimental data interpretation [8-10]. In normalized units, the numerical domain goes from -50 to 100 in the "radial" direction and from 0 to 75 in the "poloidal" direction. The region $-50 \leq x \leq 0$ corresponds to the edge, i.e. the the part of the plasma inside the Last Closed Flux Surface (LCFS). In this region, the loss terms $\left(\Sigma_{n}\right.$ and $\left.\Sigma_{T}\right)$, are set to zero in order to simulate the closure of the field lines on themselves. The LCFS is located at $x=0$ and beyond it, for $0<x \leq 50$, we have the SOL where the loss terms take their nominal values, Eqs.8-9. The last part of the numerical domain, $50<x \leq 100$, represents the wall shadow where the connection length is significantly reduced. In order to simulate this effect, in this region we increased the loss terms by a factor 20 (this is equivalent to decreasing the connection length to the solid surfaces by a realistic geometrical factor). The total domain in real space covers roughly $25 \mathrm{~cm}$ in the "radial" direction and $12.5 \mathrm{~cm}$ in the "poloidal".

All the simulations are performed with a grid of 512 points in $x$ and 256 in $y$, which assures a sufficient resolution for the turbulent structures. Furthermore, our results are not sensitive to changes in the size of the numerical box. The data we analyse are collected after the simulations enter a statistically steady state, so that spurious initial transients are not taken into account. In addition, the simulations last several thousands of turbulence correlation times (which are of the order of the tens of microseconds), so that our signals are sufficiently long to be amenable to be treated with statistical tools. 
TABLE I. Dimensional and dimensionless parameters for the reference case.

\begin{tabular}{|c|c|c|c|c|c|c|c|}
\hline$n_{0}\left[10^{19} \mathrm{~m}^{-3}\right]$ & $T_{0, i}[\mathrm{eV}]$ & $T_{0, e}[\mathrm{eV}]$ & $B_{0}[\mathrm{~T}]$ & $q$ & $L[m]$ & $a[m]$ & $R[m]$ \\
\hline 0.8 & 40 & 40 & 0.5 & 7 & 10 & 0.59 & 0.85 \\
\hline$\rho_{s} / R$ & $\epsilon$ & $D$ & $\mu$ & $\chi$ & $\Sigma_{n}$ & $\Sigma_{T}$ & \\
\hline $2.15 \mathrm{E}-3$ & 0.694 & $1.97 \mathrm{E}-3$ & $3.88 \mathrm{E}-2$ & $4.61 \mathrm{E}-3$ & $1.29 \mathrm{E}-4$ & $3.75 \mathrm{E}-3$ & \\
\hline
\end{tabular}

\section{NUMERICAL RESULTS}

Our database contains 29 simulations characterised by different values of the dimensionless parameters. The region of the parameter space that we explored is representative of the operating regime of the Mega Ampere Spherical Tokamak (MAST). In this context, we can identify a reference simulation (see Tab.I) which represents the "typical" L-mode MAST regime $[16,18]$, around which all the other simulations were constructed by changing one or more parameters.

Figure 1 shows in logarithmic scale the the range in which each dimensionless parameter was varied, together with the combinations relevant for our reference case (see Table I) and for ITER (for comparison). Note that all the simulations were performed with an inverse aspect ratio relevant for MAST $(\epsilon=0.69)$, so that this parameter does not appear in the figure. Apart from the aspect ratio, all the other dimensionless parameters span at least one order of magnitude, so that parameter space investigated is relevant also for other small and medium size machines (including conventional ones).

Although the output of the code is the full time dependent variation of all the scalar field evolved in Eqs.1-4, we focus here only on the decay length of the density, $\lambda_{n}$, temperature, $\lambda_{T}$ and heat flux, $\lambda_{q}$. These are obtained in the following way. We started by averaging the density and temperature fields, $n(x, y, t)$ and $T(x, y, t)$, first over the poloidal direction and then over time (during the period of statistically steady turbulence). This allowed us to find a single time independent radial profile of each field, $\langle n\rangle$ and $\langle T\rangle$. We then constructed $\lambda_{n}=\rho_{s}<n>/(d<n>/ d x)$ and $\lambda_{T}=\rho_{s}<T>/(d<T>/ d x)$, which are again functions of $x$. Note that the factor $\rho_{s}$ is used to make the decay lengths dimensional (we express them in $\mathrm{cm}$ ). The result of this procedure for the reference case is shown in Fig.2.

Clearly, the density and temperature profiles are not exponentially decaying since $\lambda_{n}$ 


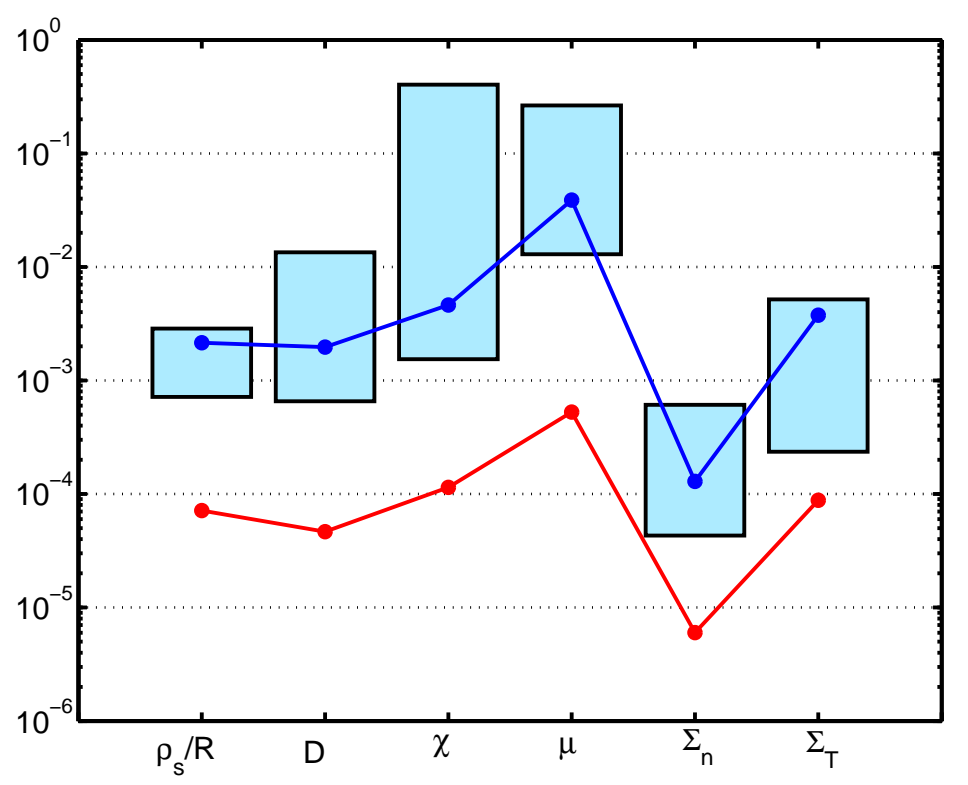

FIG. 1. The boxes represent the variation range of the dimensionless parameters in our simulation database. The upper (blue) curve tracks the parameter combination for the reference case, while the lower (red) curve representative of ITER.

and $\lambda_{T}$ have a complex radial dependence. This result is robust and appears in all our simulations. It is interesting to note that similar profiles with a local minimum after the LCFS followed by a maximum were observed experimentally in Alcator C-MOD, as reported in [19]. We interpret this peculiar shape as the result of the filament dynamics in the SOL. In particular, the maximum might be correlated with the region in which the filaments propagate ballisticaly, which leads to a relatively flat density profile.

A possible way to characterise the width of the SOL with a scalar number, is to associate it with the local minimum of the decay lengths profiles after the LCFS. This is a good (but conservative) estimate as it represents the steepest gradient in a position where the SOL is dense and hot (and hence more likely to cause problems to the divertor). In Fig.2, we marked with a circle the local minimum of the density and of the temperature. As similar minima appear in all our simulations, we identify the decay lengths with them, so that in the remaining of the paper $\lambda_{n}$ and $\lambda_{T}$ are simply scalar objects.

It is now possible to identify the relation between the decay lengths and the dimensionless parameters using statistical techniques. In particular, the data can be treated with regression 

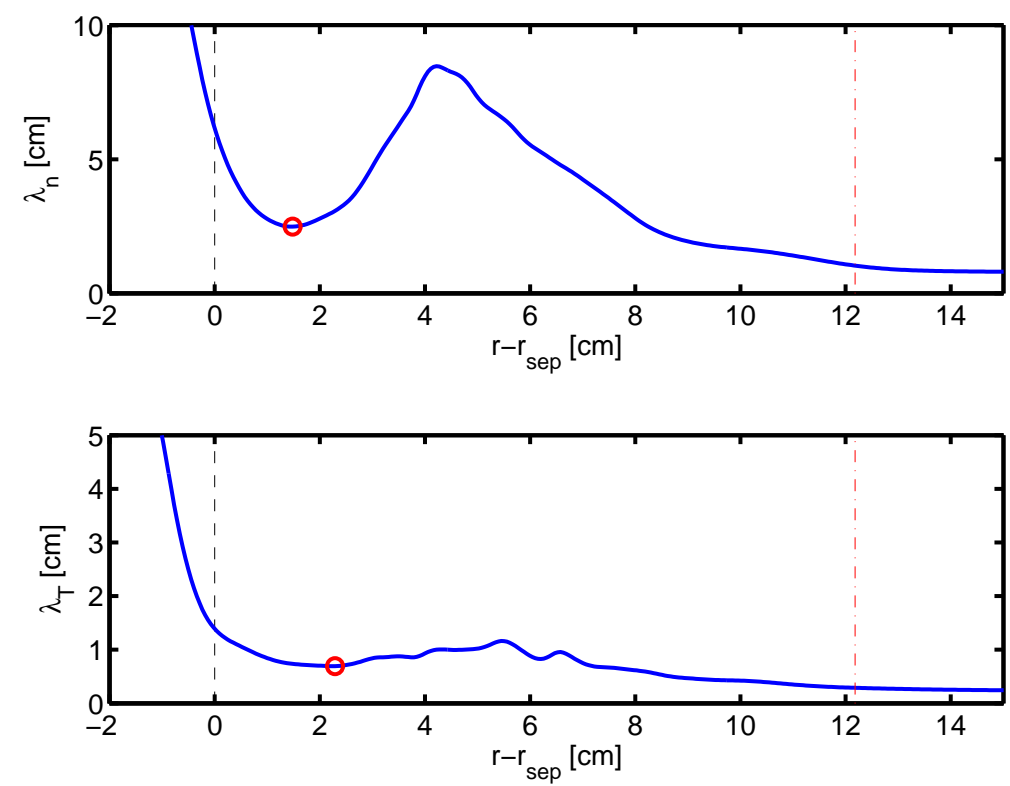

FIG. 2. Radial profiles of $\lambda_{n}$ and $\lambda_{T}$ as a function of the radial variable. Both the decay lengths and the radial variable are expressed in dimensional form (in $\mathrm{cm}$ ). The vertical thin dashed and the dash-dot lines indicate the position of the LCFS and of the wall shadow, respectively. The red circles show the position of the local minimum used to characterise the profile. The inner and outer boundaries of the computation domain of this simulation are ouside the figure at $r-r_{s e p}=-9.13 \mathrm{~cm}$ and $r-r_{\text {sep }}=18.27 \mathrm{~cm}$.

analysis by assuming the following form for a generic decay length:

$$
\frac{\lambda_{f}}{R}=\alpha\left(\frac{\rho_{s}}{R}\right)^{\beta_{1}} D^{\beta_{2}} \chi^{\beta_{3}} \mu^{\beta_{4}} \Sigma_{n}^{\beta_{5}} \Sigma_{T}^{\beta_{6}}
$$

In the calculation, we removed the statistically irrelevant dimensionless parameter using the Student's t-test and repeated the regression without them [20] (for this reason the viscosity dependence is not present in the following expressions). The resulting scaling laws for the decay length are:

$$
\begin{aligned}
& \frac{\lambda_{n}}{R}=1.04 \exp ( \pm 0.29)\left(\frac{\rho_{s}}{R}\right)^{1.09 \pm 0.07} D^{0.71 \pm 0.04} \Sigma_{n}^{-0.73 \pm 0.04} \Sigma_{T}^{-0.19 \pm 0.03} \\
& \frac{\lambda_{T}}{R}=1.91 \exp ( \pm 0.51)\left(\frac{\rho_{s}}{R}\right)^{1.07 \pm 0.12} D^{0.31 \pm 0.18} \chi^{0.14 \pm 0.12} \Sigma_{n}^{-0.10 \pm 0.07} \Sigma_{T}^{-0.52 \pm 0.07} .
\end{aligned}
$$

Both regressions give a good coefficient of determination, $\mathrm{R}^{2}$ (not to be confused with the major radius). For both the density and temperature decay lengths, $\mathrm{R}^{2}$ is 0.98 . This is further confirmed by Fig.3, which shows the actual data (obtained from the simulations) against the value computed from Eqs.11 and 12. 

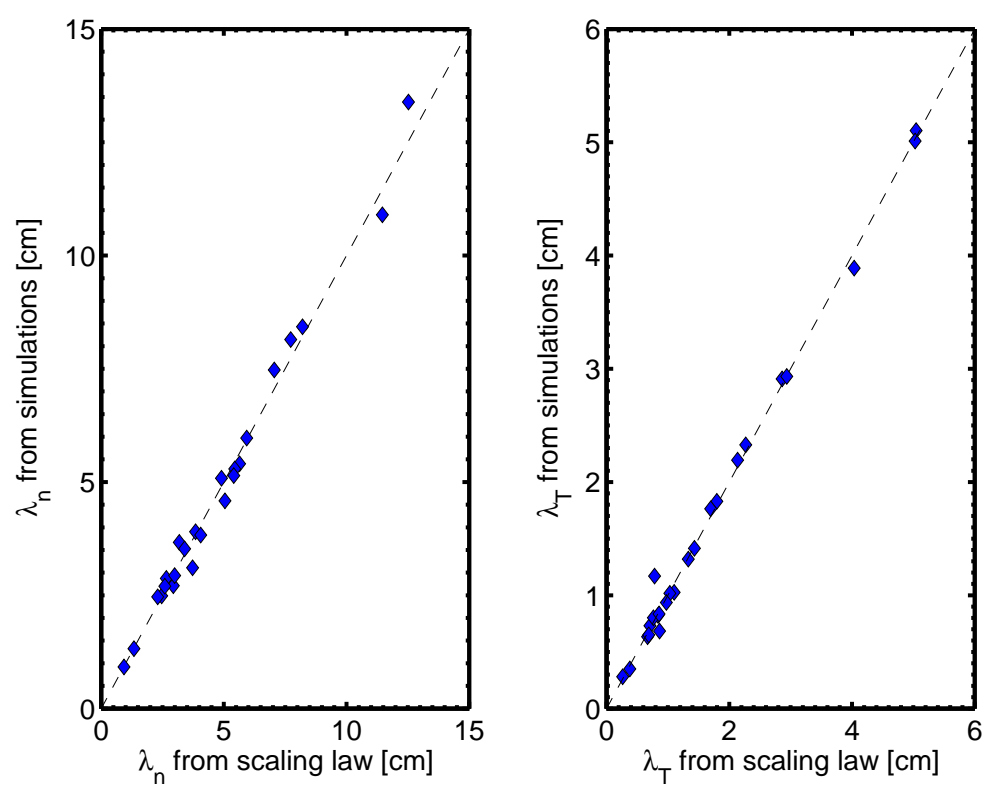

FIG. 3. The decay lengths $\lambda_{n}$ and $\lambda_{T}$, obtained from the simulations, plotted against the value computed from Eqs.11 and 12. A 45 degree dashed line is added to guide the eye.

We can now evaluate also the decay length of the parallel heat flux, $\lambda_{q}$, which is the relevant quantity to determine the target heat loads. Combining Eqs.1 and 2, we can find an expression for the evolution of the thermal power density (identified with the pressure), from which we find that $\nabla_{\|} q_{\|}=\frac{3}{2}\left(\Sigma_{T}+\Sigma_{n}\right) n T$, where $q_{\|}$is the heat flux flowing in the parallel direction. We now take $\lambda_{q} \equiv \rho_{s} \int \bar{\nabla}_{\|} q_{\|} d x /\left[3 / 2\left(\Sigma_{T}+\Sigma_{n}\right) p_{s e p}\right]$, where the overline represents poloidal average and $p_{s e p}$ is the normalized pressure at the separatrix (note that in the above expression $\lambda_{q}$ is dimensional since it is multiplied by the Larmor radius). This integral definition is well suited to capture the power deposition and it is therefore preferred to the one we used for $\lambda_{T}$ and $\lambda_{n}$. Applying regression analysis, we obtain:

$$
\frac{\lambda_{q}}{R}=2.08 \exp ( \pm 0.16)\left(\frac{\rho_{s}}{R}\right)^{1.18 \pm 0.03} D^{0.28 \pm 0.02} \Sigma_{n}^{-0.16 \pm 0.03} \Sigma_{T}^{-0.48 \pm 0.02},
$$

which gives again $R^{2}=0.98$ (see Fig.4).

The previous expressions for the decay lengths provide useful insight in the physics that determines the width of the SOL. In particular, we see that the interchange drive of the turbulence, i.e. $\rho_{s} / R$, broadens the $\mathrm{SOL}$ as all the $\lambda \mathrm{s}$ show an almost linear dependence on it. A positive, albeit weaker, dependence is also found in the dissipative parameters which contribute to spread the turbulent structures as they propagate. Conversely, the loss terms provide a mechanism that narrows the SOL. Naturally, the density decay length is 


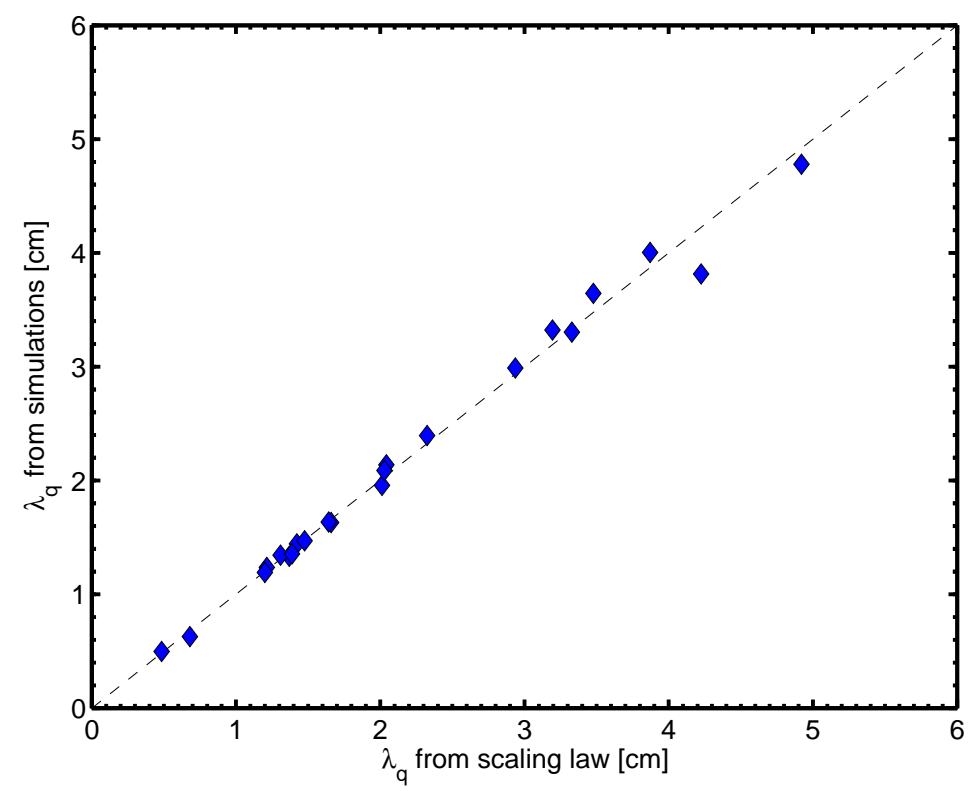

FIG. 4. The decay length $\lambda_{q}$, obtained from the simulations, plotted against the value computed from Eq.13. A 45 degree dashed line is added to guide the eye.

reduced more effectively by the density loss term, but also the temperature loss term gives a contribution (the temperature decay length shows the opposite trend).

\section{A. Scalings with measurable parameters}

While Eq.11-13 are the natural way to express how the decay lengths behave as a function of the parameters of the model, they still remain relatively obscure. In particular, it is useful to explicitly relate the $\lambda$ s to measurable quantities such as the magnetic field or the edge density. This can be straightforwardly done by replacing in the scalings, Eqs.11-13, the definition of the dimensionless parameters, Eqs.5-9. Unfortunately, this procedure relies on the fact that the parallel loss terms and the dissipative parameters are properly described by $5-9$, a fact that is not beyond dispute (for example, no rigorous theory of collisional transport exists in toroidal open field line configurations). For this reason, the results of this subsection might be subject to revision if more suitable expressions for the dimensionless parameters will become available.

In the current model, the dependence of the dimensionless parameter on the measurable parameters is often non-trivial (see e.g. Eq.6 or Eq.9) so that the decay length cannot be 


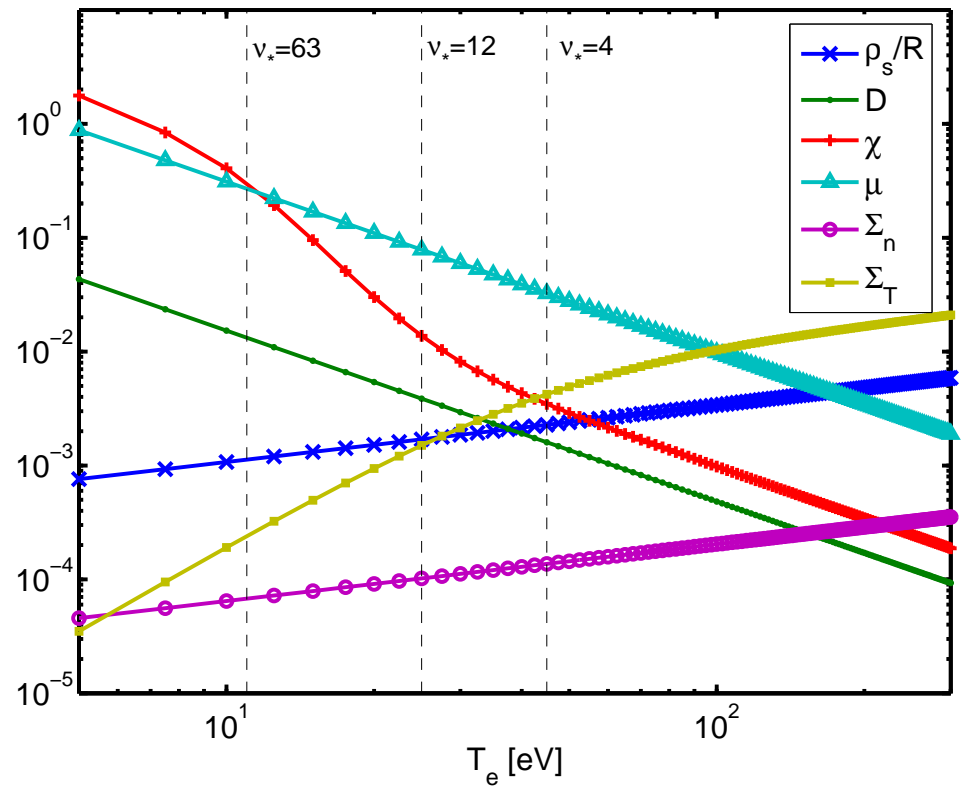

FIG. 5. Dimensionless parameters as a function of the temperature. It is assumed that $T_{i}=T_{e}$ and that the other quantities (magnetic field, safety factor,...) are the same as those in Table I. The three vertical dashed lines show the values of the temperature for which the collisionality is $\nu_{*}=63, \nu_{*}=12$ and $\nu_{*}=4$ (from left to right) corresponding to the limits of the asymptotic extrapolations.

described by a simple power law. To illustrate this complication, in Fig.5 we plotted the dimensionless parameters as a function of the temperature for the reference case (assuming that $T_{i}=T_{e}$ ). On the other hand, we observe that approximate scalings can be obtained if we restrict ourselves to certain regimes of collisionality. For arbitrary $\nu_{*}$, we have:

$$
\begin{aligned}
& \frac{\rho_{s}}{R} \sim \frac{T_{0}^{1 / 2}}{B R}, \\
& D \sim \mu \sim \frac{q^{2} n_{0}}{B T_{0}^{3 / 2}}, \\
& \Sigma_{n} \sim \frac{T_{0}^{1 / 2}}{L B} .
\end{aligned}
$$

Here we have dropped the subscripts, considered a deuterium plasma, assumed that $(1+$ $\left.1.6 q^{2}\right) \sim q^{2}$ and that the ion and electron temperature are comparable. The expression of the thermal diffusivity that we have chosen scales in a significantly different way as the 
collisionality changes. In particular, we can write:

$$
\begin{array}{ll}
\chi \sim \frac{q^{2} n_{0}}{B T_{0}^{3 / 2}} & \text { if } \nu_{*} \ll 12 \text { or } \nu_{*} \gg 63, \\
\chi \sim \frac{q^{2} n_{0}^{2} L}{B T_{0}^{7 / 2}} & \text { if } 12<\nu_{*}<63 .
\end{array}
$$

In order to derive Eq.18 we approximated $2.33+63 /\left[1+\left(63 / \nu_{*}\right)^{2}\right]$ with $\nu_{*} / 2$. This procedure in not rigorous but it allows to bridge the asymptotic regimes and obtain a rough scaling for the intermediate collisionalities. Also the parallel loss term $\Sigma_{T}$ has a different behaviour depending on $\nu_{*}$ :

$$
\begin{aligned}
& \Sigma_{T} \sim \frac{T_{0}^{1 / 2}}{B L} \quad \text { if } \nu_{*} \ll 4, \\
& \Sigma_{T} \sim \frac{T_{0}^{5 / 2}}{n_{0} B L^{2}} \quad \text { if } \nu_{*} \gg 4 .
\end{aligned}
$$

Note that the previous expressions together with the definition of $q_{\|}$above Eq.13 allow for a smooth passage from sheath to conduction limited regime as the collisionality changes.

\section{B. Heat flux decay length}

We can now derive the scalings of $\lambda_{q}$ with the measurable parameters (we are assuming $L \sim q R):$

$$
\begin{array}{ll}
\lambda_{q} \sim q^{1.18} n_{0}^{0.28} T_{0}^{-0.14} B^{-0.83} R^{0.44} & \text { if } \nu_{*} \ll 4, \\
\lambda_{q} \sim q^{1.65} n_{0}^{0.75} T_{0}^{-1.08} B^{-0.84} R^{0.91} & \text { if } \nu_{*} \ll 4 .
\end{array}
$$

It is well known that the standard approximate expression for the connection length, $L_{\|} \approx \pi q R$, is not accurate for spherical tokamaks. However, what counts in our expressions is the typical parallel length of the filaments, which reasonably scales like the pitch angle of the magnetic field (represented by $q$ ) and with the machine size (i.e. $R$ ) for every machine configuration. Hence, the approximation $L \sim q R$ is valid for both conventional and spherical tokamaks.

Unfortunately, a direct comparison with experimental scaling laws is rather difficult, since the latter are usually expressed in terms of engineering parameters such as the line averaged density and the power crossing the separatrix. As these are not control parameters for ESEL, the only possible approach is to try to relate them to the edge quantities that 
appear in Eqs.21-22 with simple models. As a working hypothesis, we assume that the edge density can be replaced with its line averaged value. This implies that the density peaking is assumed to be independent from the other parameters contained in the numerical scaling laws. With a procedure similar to the one described in [21], we relate the edge temperature with the power crossing the separatrix, which can be obtained self-consistently from our model:

$$
P_{S O L} \approx 2 \pi R \frac{\epsilon}{q} \int Q_{\|} d r
$$

where $Q_{\|}$is the total energy flux in the parallel direction (in the following we will approximate $Q_{\|}$with the heat flux, since the kinetic energy flux is negligible compared to it). We therefore have that:

$$
\int Q_{\|} d r \approx \frac{3}{2}\left(\Sigma_{n}+\Sigma_{T}\right)\left(\frac{p_{\text {sep }}}{n_{0} T_{0}}\right) n_{0} T_{0} \Omega_{i} L \lambda_{q}
$$

where $p_{\text {sep }} /\left(n_{0} T_{0}\right) \sim \chi^{0.39} \Sigma_{n}^{-0.29}$ is obtained from regression analysis applied to our database. Using 23 and 24, we can express the temperature as a function of the power and consequently $\lambda_{q}$ as a function of the power:

$$
\lambda_{q} \sim q^{1.52} n_{0}^{0.7} P_{S O L}^{-0.25} B^{-1.03} R^{0.91},
$$

in the $\nu_{*} \ll 4$ limit and:

$$
\lambda_{q} \sim q^{2.24} n_{0}^{2.28} P_{S O L}^{-1} B^{-1.78} R^{2.46}
$$

when $\nu_{*} \gg 12$.

An interesting feature of the scaling laws that we obtained is that they are able to reproduce qualitatively and in some cases quantitatively experimental results obtained at low collisionality $[22,23]$. In particular, we find a positive dependence on the safety factor and a negative on the magnetic field (thus indicating an inverse dependence on the plasma current). If we compare the L-mode experimental scaling in Ref.[23] with Eq.25, we find agreement between the exponents the magnetic field ( -0.8 versus -1.03$)$, and for the safety factor (1.14 versus 1.52). It is worth noting the lack of machine size dependence claimed in the experimental scaling laws is not retrieved. The numerical density dependence is relatively weak and it would be difficult to capture it in the regression analysis of Ref.[23] since the experimental density was varied roughly a factor 2. Note that recent scaling laws obtained with MAST data [24] and using the technique discussed in Refs.[22, 23] show an edge density 
dependence similar to ours (0.65 versus 0.7$)$. Finally, the decay length depends weakly on the power both in experimental and in the numerical scaling. However, the dependence is inverse in the two cases (i.e. positive for the experimental scaling).

It is worth noticing that similar numerical calculations performed for NSTX with the code SOLT [21] found that $\lambda_{q}$ increases with power, in apparent contradiction with our results. However, differently from our approach, the dissipative parameters in [21] are constants which do not depend on any edge quantity (also the parallel loss terms are different, but we do not discuss this difference here). If use the same assumption and repeat the calculations that led to Eq.25 and 26, we find $\lambda_{q} \sim q^{0.65} B^{-0.5} P_{S O L}^{0.16} n_{0}^{-0.16} R^{0.14}$ and $\lambda_{q} \sim q^{0.875} B^{-0.61} P_{S O L}^{-0.25} n_{0}^{0.58} R^{1.2}$ for the low and high collisionality cases respectively. Interestingly, the low collisionality scaling shows a positive power dependence, consistent with the results in [21]. In addition, these alternative expressions can be used to comment the effect of different dissipative models on our results. It is clear that the inverse dependence on the plasma current is a rather robust feature of the ESEL simulations, together with a weak power dependence (the sign of which can, however, change). On the other hand, the machine size scaling seems to depend on the particular form of the dissipative parameters (note that the low collisionality expression for $\lambda_{q}$ with constant $D, \mu$ and $\chi$ has a close resemblance to the scaling in [23], even for the $R$ dependence).

\section{Density and temperature decay lengths}

Moving to the density and temperature decay lengths, we find:

$$
\begin{array}{ll}
\lambda_{n} \sim q^{2.34} n_{0}^{0.71} T_{0}^{-0.98} B^{-0.88} R^{0.83} & \text { if } \nu_{*} \ll 4, \\
\lambda_{n} \sim q^{2.53} n_{0}^{0.9} T_{0}^{-1.36} B^{-0.88} R^{1.02} & \text { if } \nu_{*} \gg 4,
\end{array}
$$

and

$$
\begin{array}{ll}
\lambda_{T} \sim q^{1.52} n_{0}^{0.45} T_{0}^{-0.55} B^{-0.9} R^{0.55} & \text { if } \nu_{*} \ll 4, \\
\lambda_{T} \sim q^{1.63} n_{0}^{0.59} T_{0}^{-0.73} B^{-0.9} R^{0.69} & \text { if } 4 \ll \nu_{*} \ll 12, \\
\lambda_{T} \sim q^{2.18} n_{0}^{1.11} T_{0}^{-1.77} B^{-0.9} R^{1.21} & \text { if } 12<\nu_{*}<63 .
\end{array}
$$

Note that the range of validity of Eq.30 is very narrow (or even non existent), but we report its expression in order to clarify how the dependence on the measured parameters changes 
with the collisionality. In typical conditions, not much above $\nu_{*}=63$ diverted plasmas enter the detached regime, for which our calculation is likely to lose validity, and hence no scaling is provided (the model might be questionable also for very low collisionalities [25]).Also in this case, we have an acceptable agreement with the experimental scalings, the overall trends of which are properly captured by our expressions. In particular, the results obtained at JET with the MkIIA divertor [26] give an exponent for $q$ equal to 1.3 and for $B$ equal to -0.8 for both $\lambda_{n}$ and $\lambda_{T}$. In this case, the regression was made also on the edge density and temperature. For the density decay length, the experimental exponent of $n_{0}$ is 0.5 and for $T_{0}$ is -1.2 . For the temperature decay length, the values are 0.8 and -1.4 respectively.

Finally, these result are consistent with the qualitative investigation performed in [18], in which it was observed a weak dependence of the decay length on the density and a strong dependence on the temperature and on the plasma current (modelled by increasing $q$ and $L$ at the same time).

\section{SUMMARY AND CONCLUSIONS}

In this work, we presented numerically derived power law scalings for the density, temperature and heat flux decay lengths. Our results are obtained from a regression analysis of a database of 29 simulations of plasma turbulence in the SOL performed with the code ESEL. We expressed the SOL width in terms of the dimensionless parameters that govern the problem and in terms of experimentally measurable parameters.

The parameter space investigated is directly relevant for MAST plasmas, although it is not exclusive to this machine. In other words, also other small or medium size conventional tokamaks are characterized by dimensionless parameters in the range we investigated. Our simulation database, however, included only tight aspect ratio cases, so that we could not derive a scaling with $\epsilon$. Fortunately, this does not affect the validity of our results since $\epsilon$ does not depend on the measurable parameters used in Eqs.21-31 (the $R$ dependence is cancelled by a). In particular, machines with a conventional aspect ratio would still follow Eq.11-13 (and the expressions derived from them) but they would have a different constant factor in front of them [ $\alpha$ in Eq.10]. Future work will be devoted to the extension of the present analysis to a wider parameter space which could include larger machines.

It is worth noting that while the regression with respect to the dimensionless parameters 
presented in Sec.III is a rigorous procedure, the results shown in Sec.IIIA rely on the exact form of the dissipative and loss terms. As these are modelled with neoclassical theory and ad hoc assumptions for the parallel dynamics (both of which are reasonable, but certainly improvable), the scaling laws with respect to the upstream density and temperature are subject to a certain margin of error.

Nevertheless, some of the exponents of our power laws for the decay lengths are in agreement with experimental results. Although the values are not always identical (but very similar for $q$ and for $B$ ), most of the trends are properly captured. In particular, an inverse dependence of $\lambda_{q}$ on the plasma current and a weak scaling with the power crossing the separatrix are robust features of the interchange paradigm employed in our study. This suggests that the theoretical model used can reproduce the dominating mechanisms that determine the outboard midplane SOL width. At first sight this is surprising since Eq.1-4 are derived under several simplifying assumptions, among which the absence of neutrals or impurities. In addition, the model neglects the effect of the drift waves, of hot ions and it simulates the parallel dynamics with ad hoc terms. Our results suggest that these effects play only a secondary role in the determination of the upstream SOL width, although they are known to be relevant for other exhaust problems. For example, the strong magnetic shear in the proximity of the X-point might tear the filaments apart, thus changing the dominant perpendicular transport mechanism. Furthermore, neutral particles and atomic physics have a major role in the divertor region.

To partially mitigate the concerns regarding the appropriateness of this simplified $2 \mathrm{D}$ approach, it is worth noting that it was able to reproduce the features of midplane transport in several comparisons with experimental data. The ESEL code was positively tested on TCV [8], JET [9] and MAST [10] discharges. In addition, a similar code (SOLT) employing the same philosophy showed good agreement with NSTX [21] and Alcator C-MOD [27].

Finally, at low collisionality our expressions describe a certain machine size dependence and a (small) negative exponent for the power crossing the separatrix. These results are in contradiction with the most recent experimental scaling laws. Possible reasons for this discrepancy, beyond those listed above, are the fact that the dissipative and parallel loss terms used in the simulations do not change in space and time and that the simulations were not performed in a regime that was relevant for large machines. New numerical campaigns will be needed to verify these hypotheses. 


\section{ACKNOWLEDGEMENTS}

F.M. acknowledges useful discussions with Dr. A. Scarabosio, Dr. T. Eich, Dr. J. Harrison, Dr. G. Fishpool and Dr. W. Morris. This work was funded by the RCUK Energy Programme under grant EP/I501045 and the European Communities under the contract of Association between EURATOM and CCFE. The views and opinions expressed herein do not necessarily reflect those of the European Commission.

[1] A.Loarte, B. Lipschultz, A.S. Kukushkin et al., Nucl. Fusion 47, S203 (2007).

[2] S.J. Zweben and R.W. Gould, Nucl. Fusion 25, 171 (1985).

[3] A.J. Wootton, B.A. Carreras, H. Matsumoto et al., Phys. Fluids B 2, 2879 (1990).

[4] S.J. Zweben et al., Nucl. Fusion 44, 134 (2004).

[5] Y. Sarazin and Ph. Ghendrih, Phys. Plasmas 5, 4214 (1998).

[6] S.I. Krashenninikov, Phys. Lett. A 283, 368 (2001).

[7] O.E. Garcia, V. Naulin, A.H. Nielsen and J.J. Rasmussen, Phys. Rev. Lett. 92, 165003 (2004).

[8] O.E. Garcia, J. Horacek, R.A. Pitts et al., Plasma Phys. Control. Fusion 48, L1 (2006).

[9] W. Fundamenski, O.E. Garcia, V. Naulin et al., Nucl. Fusion 47, 417 (2007).

[10] F. Militello, P. Tamain, A. Kirk et al., Submitted to Plasma Phys. Control. Fusion (2012).

[11] V. Naulin, O.E. Garcia, A.H. Nielsen, J.J. Rasmussen, Physics Letters A 321, 355 (2004).

[12] J.R. Angus, M.V. Umansky, S.I. Krashenninikov, Phys. Rev. Lett. 108, 215002 (2012).

[13] B.B. Kadomtsev, Sov. Phys. - J. Plasma Phys., 1, 295 (1975).

[14] J.W. Connor and J.B. Taylor, Nucl. Fusion, 17, 1047 (1977).

[15] T.C. Luce, C.C. Petty and J.G. Cordey, Plasma Phys. Control. Fusion, 50, 043001 (2008).

[16] F. Militello and W. Fundamenski, Plasma Phys. Control. Fusion 53, 095002 (2011).

[17] O.E. Garcia, N.H. Bian, W. Fundamenski, Phys. Plasmas 13, 082309 (2006).

[18] F. Militello, W. Fundamenski, V. Naulin, A.H. Nielsen, Plasma Phys. Control. Fusion 54, $095011(2012)$.

[19] B. LaBombard, J.W. Huges, D. Mossessian et al., Nucl. Fusion 45, 1658 (2005).

[20] W. Fundamenski, T. Eich, S. Devaux et al., Nucl. Fusion 51, 083028 (2011).

[21] J. R. Myra, D. A. Russell, D. A. DIppolito et al., Phys. Plasmas 18, 012305 (2011). 
[22] T. Eich, B. Sieglin, A. Scarabosio et al., Phys. Rev. Lett. 107, 215001 (2011).

[23] A. Scarabosio, T. Eich, A. Herrmann, B.Sieglin, Paper P1-33 in Proceedings of the 20th International Conference on Plasma Surface Interactions, Aachen, May 2012.

[24] J.R. Harrison, G.M. Fishpool and A. Kirk, Paper P1-19 in Proceedings of the 20th International Conference on Plasma Surface Interactions, Aachen, May 2012.

[25] O.E. Garcia, J. Horacek, R.A. Pitts et al., Nucl. Fusion 47, 667 (2007).

[26] W. Fundamenski, Fusion Science and Technology 53, 1023 (2008).

[27] D. A. Russell, D. A. DIppolito, J. R. Myra et al., Phys. Plasmas 19, 082311 (2012). 